\title{
Development of a Relationship between External Measurements and Reinforcement Stress

\author{
${ }^{a}$ Dept. of Civil Engineering, Queen's Univ., 58 University Ave., Kingston, ON, Canada K7L 3N6; \\ ${ }^{b}$ Dept. of Engineering, Univ. of Cambridge, Trumpington St., Cambridge, UK CB2 1PZ
} \\ Andre Brault ${ }^{\mathrm{a}}$, Neil A. Hoult*a, Janet M. Lees ${ }^{\mathrm{b}}$
}

\begin{abstract}
As many countries around the world face an aging infrastructure crisis, there is an increasing need to develop more accurate monitoring and assessment techniques for reinforced concrete structures. One of the challenges associated with assessing existing infrastructure is correlating externally measured parameters such as crack widths and surface strains with reinforcement stresses as this is dependent on a number of variables. The current research investigates how the use of distributed fiber optic sensors to measure reinforcement strain can be correlated with digital image correlation measurements of crack widths to relate external crack width measurements to reinforcement stresses. An initial set of experiments was undertaken involving a series of small-scale beam specimens tested in three-point bending with variable reinforcement properties. Relationships between crack widths and internal reinforcement strains were observed including that both the diameter and number of bars affected the measured maximum strain and crack width. A model that uses measured crack width to estimate reinforcement strain was presented and compared to the experimental results. The model was found to provide accurate estimates of load carrying capacity for a given crack width, however, the model was potentially less accurate when crack widths were used to estimate the experimental reinforcement strains. The need for more experimental data to validate the conclusions of this research was also highlighted.
\end{abstract}

Keywords: Assessment, crack widths, Digital Image Correlation, distributed fiber optic strain sensors, reinforced concrete structures

\section{INTRODUCTION}

Many of the reinforced concrete infrastructure assets (e.g. bridges, dams, and tunnels) that were built in the post-war construction booms of the $1950 \mathrm{~s}$ and $60 \mathrm{~s}$ are coming to the end of their theoretical design lives. However, there are limited financial resources with which to maintain, repair, and replace these structures. Thus an approach that would allow safety critical structures to be identified so that they can be made an investment priority is needed. In reinforced concrete, the stress in the steel reinforcement is of critical importance since if the reinforcement stress is too high this could be indicative of overloading or even imminent failure of the structure. The stress in the reinforcement is typically highest at the location of cracks in the concrete since cracks develop due to localized concentrations of tensile stress. Measuring the reinforcement strains, and thus the stress, is not possible within existing reinforced concrete infrastructure; however cracks are monitored as part of visual inspections required for many critical infrastructure assets. If a technique could be developed for measuring crack widths during visual inspections and an approach for relating these crack measurements to reinforcement stress could also be developed, this would enable safety critical structures to be identified.

Calvi ${ }^{1}$ has proposed a method for using crack widths obtained during visual inspections to estimate the reinforcement stress. Although Calvi undertook an initial validation of his model using an experimental database, one of the challenges associated with validating this approach is correlating the crack width with the maximum strain in the reinforcement. In his experimental campaign, Calvi did this by debonding the reinforcement in the area of the crack, which created a region of constant strain. However, in actual structures it is not possible to know where cracks will develop exactly nor is it feasible to provide debonded regions to enable the measurement of strain at the exact location of the crack. Without prior knowledge as to where a crack will form, consistently locating a conventional strain gauge on the reinforcement at the future location of the crack is impossible. Other researchers ${ }^{2,3}$ have required the use of numerous strain gauges installed along the reinforcement with the assumption that one of these gauges will

Sensors and Smart Structures Technologies for Civil, Mechanical, and Aerospace Systems 2015,

edited by Jerome P. Lynch, Kon-Well Wang, Hoon Sohn, Proc. of SPIE Vol. 9435, 943519

(C) 2015 SPIE · CCC code: $0277-786 X / 15 / \$ 18 \cdot$ doi: $10.1117 / 12.2175830$

Proc. of SPIE Vol. 9435 943519-1 
capture the strain at the formation of the crack. This technique has numerous problems including the large quantity of strain gauges and wires required, which may themselves affect the bond between the reinforcement and the surrounding concrete, and the cost of the instrumentation and the installation. Measurement of the crack width is also difficult. Current approaches to crack width measurement in the field typically involve the use of a crack gauge or a telltale, both of which have to be read by the inspector and are thus subject to significant measurement error ${ }^{4}$.

Recent advances in sensor technologies may enable the problems associated with both strain and crack width measurement to be overcome. Distributed fiber optic sensors (FOS) enable the strain to be measured over gauge lengths equivalent to conventional strain gauges (e.g. $5 \mathrm{~mm}$ ) but at the same time allowing strain to be measured along the full length of a fiber optic cable. This eliminates the need for a number of strain gauges and their wires while still enabling the maximum strain to be measured at any location on the reinforcement. Digital Image Correlation (DIC) uses images taken by digital cameras to track displacement fields during the loading of a structure. Using DIC software the crack width can be measured with an accuracy that is several orders of magnitude higher than conventional crack sensors depending on the setup. Thus, DIC allows the processing of crack width data following any test as long as the crack develops within the camera's field of view, and distributed FOS will allow reinforcement strain measurements to be captured at the crack location. This allows FOS measured strains at crack locations to be correlated with the corresponding DIC measured crack widths.

The ultimate goal of the research is to develop a technique for measuring crack widths using DIC and use these measurements to determine the reinforcement stress. In the current research an experimental investigation has been undertaken using both distributed DIC and FOS measurements in an attempt to relate crack width to reinforcement stress. These results are then compared to a proposed model. The objectives of this study are to: (i) develop a method for measuring crack widths and reinforcement strains in reinforced concrete beams using distributed sensors, (ii) determine the relationships between crack width and reinforcement strain in beams with varying reinforcement properties, and (iii) compare the experimental results to a previously developed analytical model. This paper is divided into five sections: Section 2 provides background information on the sensor technologies and the analytical model used to relate crack width to reinforcement strain. Section 3 outlines the experimental and analytical procedures used in this research program. Section 4 presents the results of the research, and Section 5 presents the general conclusions of the research and opportunities for future work.

\section{BACKGROUND}

\subsection{Fiber Optic Strain Sensing}

There are two main types of FOS technologies: discrete and distributed. Discrete technologies provide readings at a limited number of defined points along the fiber optic cable while distributed technologies enable the entire fiber optic cable to be used as a series of sensors. Fiber Bragg Gratings (FBG) have shown comparable accuracy to conventional strain gauges but like conventional strain gauges only have the capability to measure strain readings at discrete locations. 5 . Distributed FOS systems are based on measuring light reflected back through the fiber optic cables (backscattered light) to the FOS analyzer unit. There are three main types of backscattered light: Raman, Brillouin, and Rayleigh. Raman sensing systems can only be used to measure temperature and as such do not apply to the current research. Brillouin systems have been used to measure strain in a number of civil engineering applications including reinforced concrete beams ${ }^{6}$. However, the major limitation with Brillouin systems for this application is that the spatial resolution is approximately one meter, which is too large to measure the strains at the crack location ${ }^{7}$. As such, the backscatter sensing technology used in the current research is based on Rayleigh Backscatter, which occurs due to interactions between the propagated light pulse and the silica density composition of the fiber at each point along its length. The light pulse reflects off of these imperfections along the fiber length and scatters portions of the light spectrum back to the analyzing unit ${ }^{8}$. When the fiber is strained the distance between these imperfections changes, which in turn changes the backscattered light spectrum measured at that location. By comparing the backscatter spectrum measured in sequential scans to one from a reference scan, strain and temperature measurements can be calculated based on change in light frequency along the full fiber length. Using this technology, strain and temperature readings have been measured with an accuracy of approximately 1 microstrain or 0.1 degrees Celsius with a spatial resolution of 10 millimeters over fiber lengths spanning up to 70 meters long. ${ }^{9}$ However, strain and temperature readings are not independent, and therefore require the temperature to remain constant or to be measured to distinguish and correct for temperature induced strains. In the current research the testing was conducted at constant temperature to eliminate these effects. 
Previous researchers have used Rayleigh based distributed FOS to measure strains in reinforced concrete structures. For example, Villalba and Casas ${ }^{10}$ used this technology to detect the presence of cracks in a slab specimen under load even before the cracks were visible. Regier ${ }^{11}$ evaluated different adhesive and fiber coating combinations based on the accuracy of the measurement produced when compared with electrical resistance strain gauge readings. The combinations of polyimide fiber with Loctite 4851 cyanoacrylate adhesive was determined to be the combination that led to the most accurate strain readings for internal steel reinforcement (and thus will be employed in the current study). Regier and Hoult ${ }^{12}$ used the system to measure the distributed strain profile of a reinforced concrete bridge during a load test. While Regier and Hoult ${ }^{13}$ used the technique to detect localized reinforcement deterioration including investigating changes in internal reinforcement strain along the full length of the bar. However, the current investigation is the first that the authors know of to attempt to correlate internal FOS strain measurements with external crack measurements.

Bare fiber optic cables are inappropriate for civil engineering applications and are thus covered in a buffer or coating to increase their strength and durability. When a fiber is coated there is a compromise between the transfer of strain and temperature from the material being monitored to the fiber optic cable core where the strain readings are ultimately measured. The fiber optic cable used in this research has a polyimide coating. Polyimide coated fibers have good strain and temperature transfer between the polyimide coating and the fiber core, however durability and susceptibility to large strain gradients are issues ${ }^{13}$.

\subsection{Digital Image Correlation}

DIC is the use of digital images to measure displacements of regions of interest in images of a deformed object as compared to its undeformed state (i.e. the reference image). In structural engineering applications this usually entails taking images of a structure before and during loading to determine displacements and in some cases strains.

DIC has been used by a number of researchers for the measurement of crack widths in reinforced concrete. For example, Lecompte et al. ${ }^{14}$ used DIC on a prestressed beam test to detect cracking while Destrebecq et al. ${ }^{15}$ employed DIC to detect the development of cracks in a reinforced concrete beam tested in the laboratory. Ferreira et al. ${ }^{16}$ used DIC to measure crack widths in notched reinforced concrete beam tests. And in a recent study Jason et al. ${ }^{17}$ used DIC to measure horizontal crack width opening in the constant moment region of beams to better understand the reinforcement bond stress. However, while many researchers have used DIC to measure crack widths and to attempt to understand reinforcement stress, the focus of the current study is the linking of DIC measured crack widths with distributed FOS reinforcement strain measurements in an attempt to understand the relationship between reinforcement stress and crack width.

In the current research the DIC measurements were obtained using a software package called GeoPIV ${ }^{18}$. GeoPIV was initially developed for geotechnical monitoring but has since been applied to measuring bridge displacements ${ }^{19}$ as well as surface strain in axial ${ }^{20}$ and bending members ${ }^{21}$.

\subsection{Correlation of Crack Width to Reinforcement Strain Model}

Calvi ${ }^{1}$ has developed the "Assessment of Cracked Reinforced Concrete" (ACRC) model, which relates measured crack widths to estimated reinforcement stresses. The ACRC model's primary inputs are crack width, crack slip, and crack orientation, as well as the material and geometric properties of the section, which are used to develop an estimate of the global stress and strain state in a reinforced concrete element.

In order to generate a compatibility relationship in the ACRC model, Calvi ${ }^{1}$ developed a relationship between reinforcement strain, $\varepsilon_{s}$, and external concrete crack width, $w$, using previously generated analytical steel-concrete bond relationships from Shima et al. ${ }^{22}$ and Maekawa et al. ${ }^{23}$. Calvi ${ }^{1}$ suggests that equation 1 can be used to estimate the strain in the reinforcement at a crack location.

$$
\varepsilon_{s}=\frac{-\beta+\sqrt{\beta^{2}+14000 S_{a, b}}}{7000}
$$


The term $\beta$ presented in equation (1) was suggested to be 2 for deformed reinforcement by Shima et al. ${ }^{22}$ based on their experimental testing but depends on bond degradation and can potentially range up to a value of 6 . The term $S_{a, b}$ is a non-dimensional reinforcement pull-out slip term that can be determined using equation (2).

$$
S_{a, b}=\frac{S_{b}}{d_{b}} K_{f c}
$$

The reinforcement pull-out slip, $s_{b}$, can be estimated using equation (3) based on the empirical relationship developed by Maekawa et. al. ${ }^{23}$ The reinforcement diameter, $d_{b}$, is given in millimeters. And $K_{f c}$ is a concrete strength adjustment factor given by equation (4).

$$
\begin{aligned}
s_{b} & =\frac{w}{2.6} \\
K_{f c} & =\left(\frac{f_{c}^{\prime}}{20}\right)^{2 / 3}
\end{aligned}
$$

Thus, in the method presented by Calvi ${ }^{1}$ if the crack width, $w$, can be measured and the concrete strength, $f_{c}^{\prime}$, is known, the reinforcement strain can be estimated. If the modulus of elasticity, $E$, and bar area, $A_{s}$, are known, the reinforcement stress can then be estimated using Hooke's Law.

\section{METHODOLOGY}

\subsection{Specimens}

Three different reinforced concrete beam specimens were tested denoted as: A, B, and C. Each beam had a total length of $840 \mathrm{~mm}$ and a loaded span of $740 \mathrm{~mm}$. The cross section for beam specimens $\mathrm{A}, \mathrm{B}$, and $\mathrm{C}$ is shown in Figure 1 . The concrete mix for each specimen was designed to have a compressive strength of approximately $30 \mathrm{MPa}$. Stirrups with a diameter of $5 \mathrm{~mm}$ at a spacing of $100 \mathrm{~mm}$ were used in all specimens in an attempt to ensure a flexural failure and avoid the development of large shear cracks during the test. The overall cross-section dimensions and the approximate effective depth of all the specimens were kept constant to isolate the effect of the different longitudinal reinforcement areas and diameters on the specimen behavior. The reinforcement ratio for specimen $\mathrm{A}$ was $1 \%$ while specimens $\mathrm{B}$ and $\mathrm{C}$ had a reinforcement ratio of $2 \%$ as indicated in Table 1 . The reinforcement bar surface area, given as the reinforcement circumference in Table 1, differs between all three specimens. The variations in reinforcement ratio and surface area were selected to enable observations to be made about the impact of these properties on the relationship between crack width and reinforcement stress.

Table 1: Specimen reinforcement geometric properties

\begin{tabular}{llll}
\hline Specimen & A $(1 \times 10 \mathrm{M})$ & B $(2 \times 10 \mathrm{M})$ & $\mathrm{C}(1 \times 15 \mathrm{M})$ \\
\hline Reinforcement area $\left(\mathrm{mm}^{2}\right)$ & 100 & 200 & 200 \\
\hline Reinforcement ratio & $1 \%$ & $2 \%$ & $2 \%$ \\
\hline Total Reinf. Circumference $(\mathrm{mm})$ & 35.5 & 71 & 50 \\
\hline Reinf. Circumference/area ratio & 0.355 & 0.355 & 0.25 \\
\hline
\end{tabular}




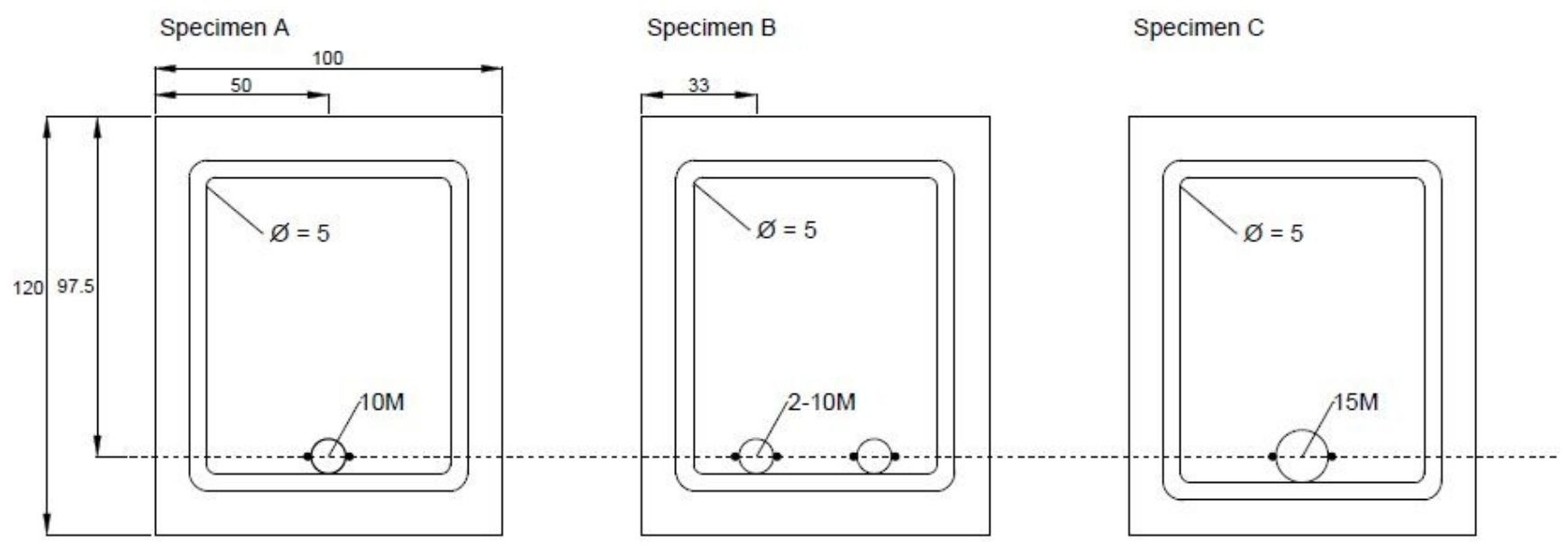

- Denotes Fiber Optic Sensors

Figure 1. Specimen dimensions ( $\mathrm{mm})$, including reinforcement specifications and fiber optic cable locations

\subsection{Instrumentation}

A distributed FOS system was used to measure reinforcement strain at the mid-height of each specimen's reinforcement bars. The fiber optic cable was a polyimide fiber bonded with a Loctite 4851 cyanoacrylate adhesive. Prior to the installation of the fiber optic cable, the reinforcement was cleaned of rust, dirt and oil with rubbing alcohol to ensure bond between the fiber and the reinforcement. One polyimide fiber was installed on each side of the reinforcement bars for all specimens. The internal fiber locations are illustrated in Figure 1.

DIC was used to measure crack widths at the mid height of the reinforcement on the surface of the beam. Both sides of each specimen were monitored using Canon Rebel T2i cameras with $180 \mathrm{~mm}$ macro lenses with a field of view of 5196 pixels by 3464 pixels. Both cameras were focused on the middle third of the beam's loaded span where flexural cracks were expected to form. A speckle pattern was applied to the middle third of each beam with black and white spray paint to add surface texture to increase the strain measurement accuracy of the GeoPIV algorithm. An example of a textured beam specimen showing the field of view of the camera is shown in Figure 2.

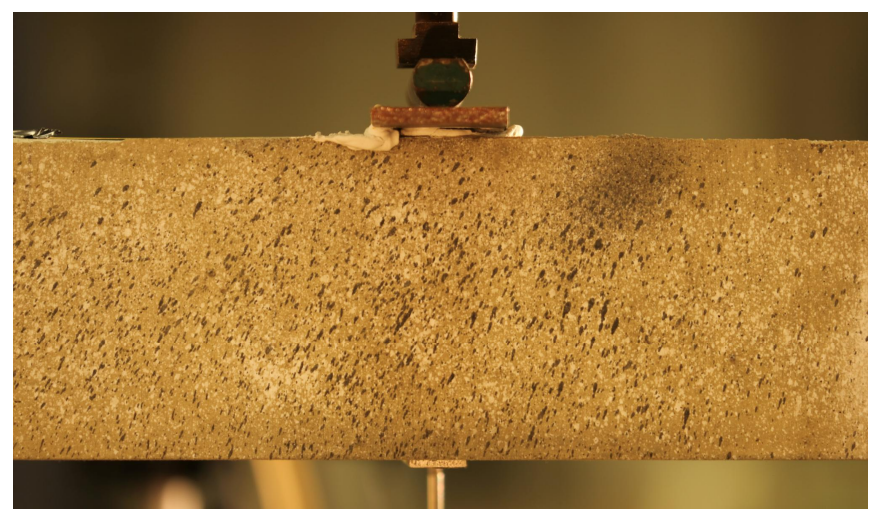

Figure 2. Example of field of view of DIC images showing speckle pattern applied to the concrete beam exterior

\subsection{Test Set-Up and Procedure}

Each beam specimen was tested in 3 point bending. The specimens were loaded at a rate of $5 \mathrm{~mm}$ per minute under displacement control. A $50 \mathrm{~mm}$ wide and $5 \mathrm{~mm}$ thick steel plate that spanned the full width of beam $(100 \mathrm{~mm})$ was placed on a thin layer of plaster on top of the beam to spread the point load out evenly over the $50 \mathrm{~mm}$ by $100 \mathrm{~mm}$ load 
pad area. Load and stroke data was acquired directly from the testing machine and logged using a data acquisition system at a rate of $10 \mathrm{~Hz}$. Fiber optic data was acquired using a LUNA OBR 4600 analyzer. DIC data was acquired from the Canon Rebel T2i images that were taken using a trigger system that stored each image on the camera's memory card. Additional lighting was provided to ensure image consistency and clarity. The full test set-up including the LUNA OBR 4600 analyzer and cameras is shown in Figure 3.

The ultimate applied point load was estimated to be approximately $20 \mathrm{kN}, 40 \mathrm{kN}$, and $40 \mathrm{kN}$ for specimens A, B, and C, respectively. Specimen A was loaded in $2 \mathrm{kN}$ increments until yielding of the longitudinal reinforcement commenced at approximately $24 \mathrm{kN}$. Specimens B and C were loaded in $4 \mathrm{kN}$ increments until initial yielding at $36 \mathrm{kN}$ and $38 \mathrm{kN}$, respectively. This loading plan provided a similar number of load stages for each specimen, and numerous matching load stages for specimen comparison (e.g. $2 \mathrm{kN}, 4 \mathrm{kN} \ldots 12 \mathrm{kN}, 16 \mathrm{kN} \ldots$ etc.).

An initial fiber optic reference scan was taken prior to each test which was then subtracted from subsequent scans to in order to provide a spectral shift measurement that was used to calculate the strain along the fiber optic cable. Fiber optic readings were taken and saved at each load stage until the test was terminated. Ten images were taken using the Canon Rebel T2i cameras prior to testing to create reference images and at each load. Multiple images were required for averaging in the analysis process to reduce errors induced by outlying images potentially caused by lighting differences or human obstruction. Lastly, observations were recorded at each stage including crack formations, crack growth, and any unexpected results or behavior.

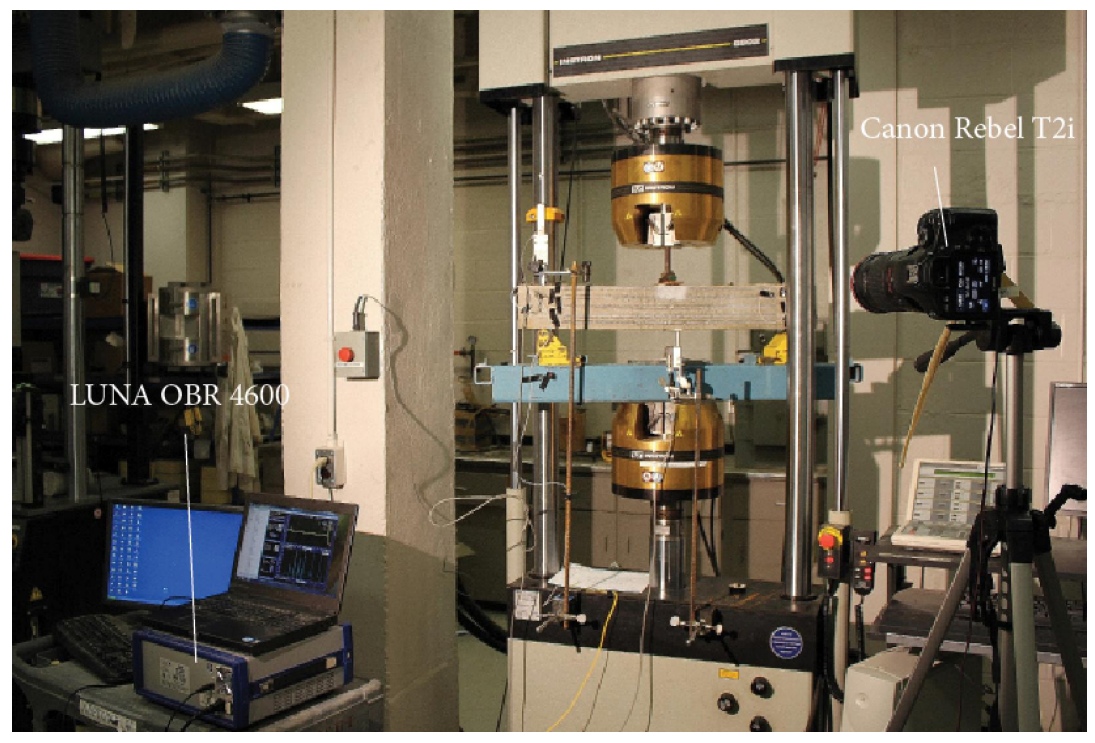

Figure 3. Test set-up including LUNA OBR 4600 and DIC camera orientation.

\subsection{Analytical Method}

The crack widths were measured using the DIC technique. This was done by tracking the movements of subsets (64 by 64 pixel areas in the image) on either side of a crack location, and converting pixel measurements into a crack width in millimeters using a scale factor. An example of the DIC subsets used on either side of a flexural crack at the level of the internal reinforcement is shown in Figure 4(a). The FOS system was used to measure the strain along the reinforcement using a gauge length of $5 \mathrm{~mm}$ and a sensor spacing of $5 \mathrm{~mm}$. An illustration of the strain distribution over the length of the reinforcement with the crack location highlighted is shown in Figure 4(b). The crack widths measured with DIC and maximum reinforcement strains measured with FOS were then used to examine the relationship between crack width and reinforcement stress as discussed in the next section. 


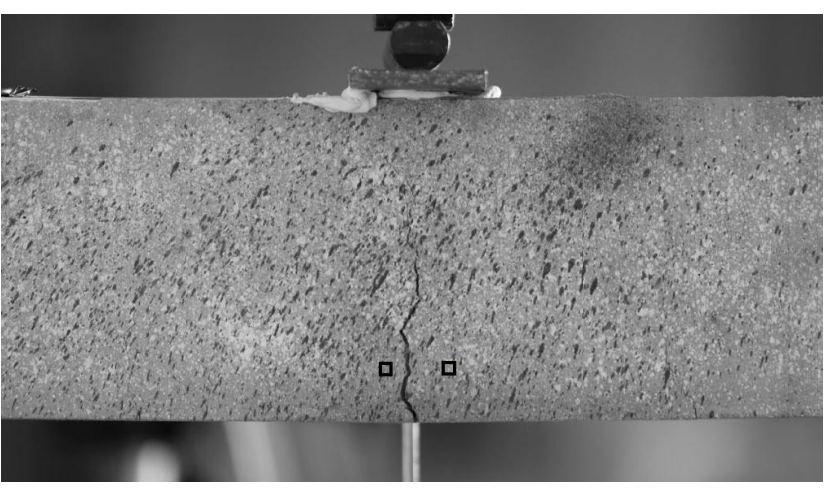

(a) DIC subsets used to measure crack width on either side of the concrete surface crack

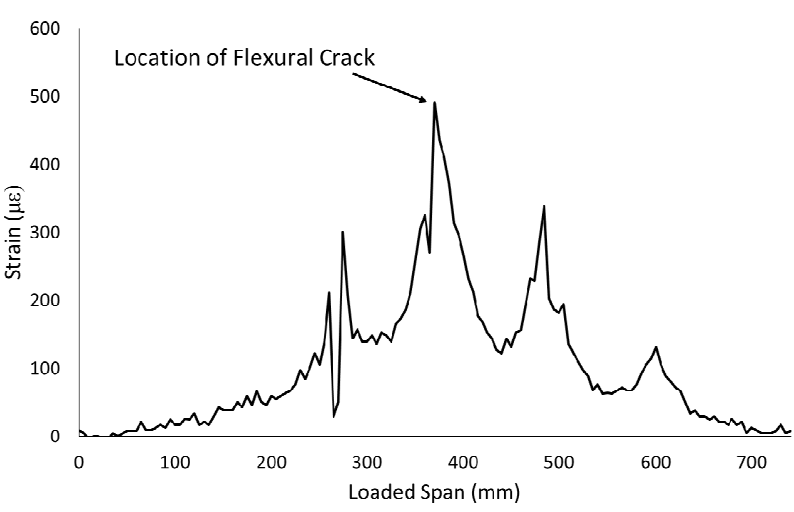

(b) Measured reinforcement strain along the loaded span for Specimen $\mathrm{A}$ at $4 \mathrm{kN}$ with the strain at the location of the concrete crack highlighted

Figure 4: Example DIC image and FOS reinforcement strain profile.

\section{RESULTS AND DISCUSSION}

In order to evaluate the accuracy of the FOS measurements at crack locations, an initial evaluation exercise was performed, the results of which are presented in Figure 5. Figure 5 shows the relationship between the maximum reinforcement strain at each specimen's crack location measured by the FOS system compared to a theoretical reinforcement strain based upon static equilibrium for increasing applied loads. The theoretical strain values were determined using reinforcement stress results from a reinforced concrete analysis software package called Response $2000^{24}$. The theoretical strain is used in this case to evaluate the accuracy of the FOS measurements because of the difficulty of acquiring maximum strain measurements with conventional techniques as previously discussed. Also plotted in Figure 5 is a 1:1 line that denotes a perfect correlation between theoretical and measured results.

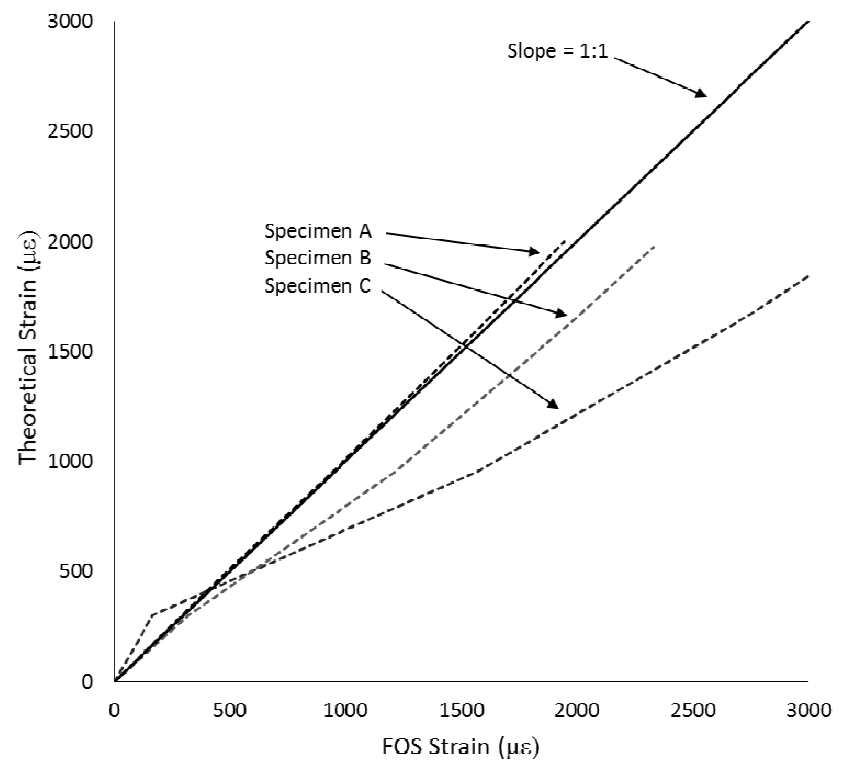

Figure 5. Theoretical reinforcement strains vs. strains measured with FOS for each Specimens A, B, and C 
One can see from Figure 5 that the FOS measurements show good visual correlation with the theoretical estimate for Specimen A. The results for Specimen B are also correlated within a reasonable level of error (15\%) given that variable properties like concrete compressive strength, steel reinforcement modulus and geometry may not have been exactly the same in the theoretical analysis and the experimental specimens. The FOS strain measurements for Specimen $\mathrm{C}$ are larger than those predicted theoretically by approximately $40 \%$. The magnitude of the difference is significant and it is not immediately clear what has caused it as debonding of the fiber optic cable or slip between the fiber and the reinforcement would both cause a reduction in measured strain rather than an increase. Part of the error could be attributed to differences between theoretical assumptions and actual specimen parameters similar to those discussed for Specimen B. However due to the magnitude of the difference it is likely that additional factors are increasing the FOS measurements for Specimen C. These may include disturbed region effects as the crack is immediately beneath the point load, which would locally increase tensile strains beneath the loading point. Additionally, only FOS measurements from one side of the reinforcement bar remained reliable throughout the test, and if the reinforcement was placed with the FOS cable oriented towards the bottom of the specimen it would measure higher tension values than expected due to the small depth of the members. The differences in strain mean that any conclusions drawn about Specimen C's behavior should be treated with caution as other mechanisms may have affected the reinforcement strain.

Figure 6 shows the relationship between maximum reinforcement strain at the crack measured by the FOS system against the crack width measured using the DIC system for Specimens A, B and C. Also plotted in Figure 6 is the strain predicted using the method proposed by Calvi ${ }^{1}$. To do this, the crack widths measured using the DIC system were inputted into equation (3) and the specimen properties were inputted into equations (2) and (4). The resulting values were then used in equation (1) to estimate the reinforcement strain using a $\beta$ value of 2 and 6 in Figures 6(a) and 6(b), respectively.

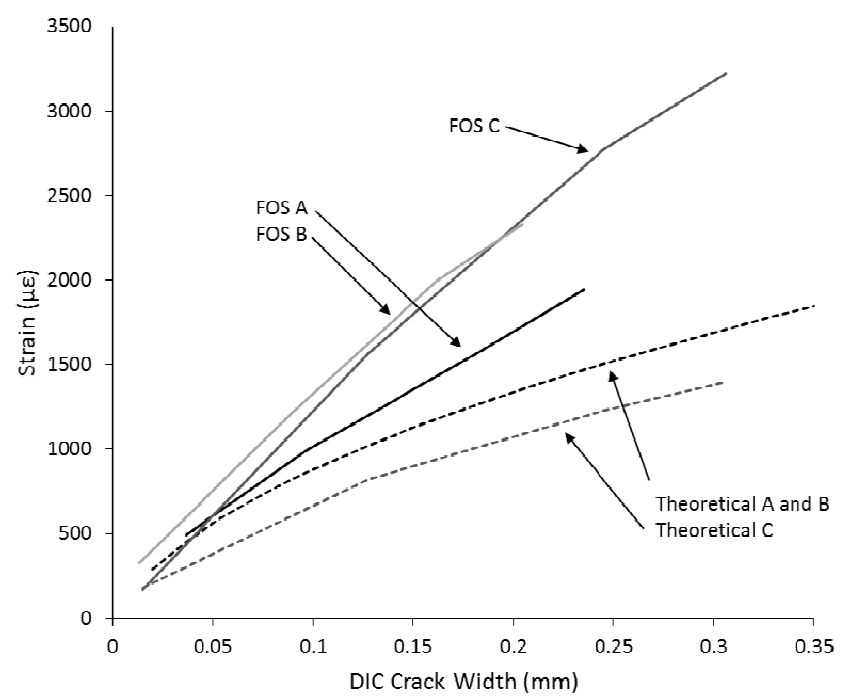

(a) $\beta=2$

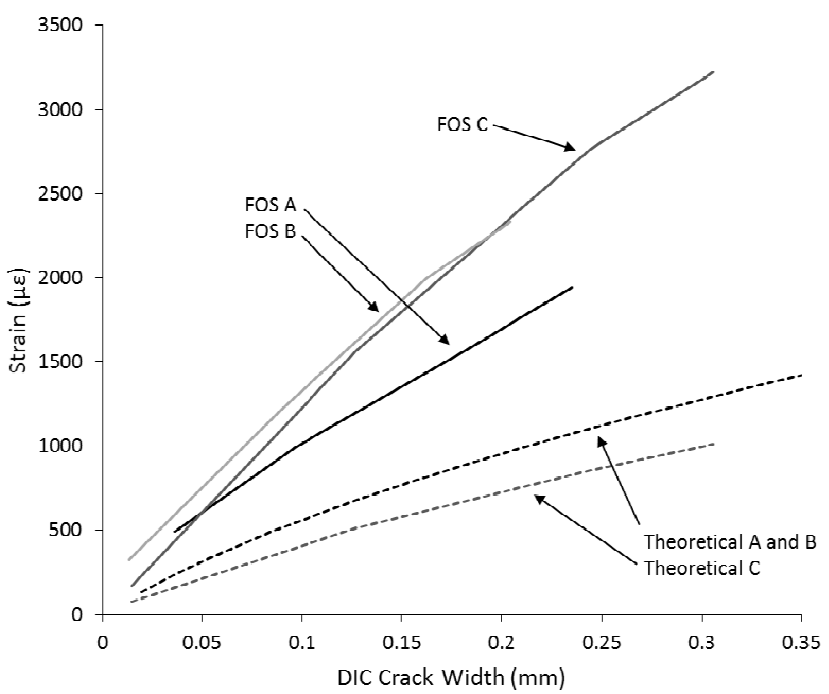

(b) $\beta=6$

Figure 6. FOS and theoretical reinforcement strain at the crack for two levels of $\beta$ vs. DIC crack width for Specimens A, B, \& C.

One can see from Figure 6 that Specimen A has the highest crack width for a given strain while Specimens B and C have very similar crack widths for a given strain. The result is an interesting one as according to Gergely and Lutz's ${ }^{25}$ pioneering work on crack width estimation, the reinforcement stress is the most important variable in the control of crack widths. Thus at a constant strain value (and thus a constant stress for a material with the same elastic modulus) one might expect the crack widths to be the same. However, Gergely and Lutz did suggest that the use of more small diameter bars would result in better crack control and so the fact that Specimen B with two 10M bars has better crack control than Specimen A with one $10 \mathrm{M}$ at a given stress level is perhaps not unexpected. Initially Specimen B also shows better crack control than Specimen C (with one 15M bar), which also seems to confirm the conclusion that multiple small bars will control cracks better than one large bar with the same overall area. However, since the Specimen $\mathrm{C}$ measured reinforcement strains were higher than expected, it is difficult to know how this relationship would have developed at the expected strain levels. 
One can also see from Figure 6(a) that the proposed approach for estimating the reinforcement strains based on crack widths and a $\beta$ value of 2 provides lower predictions of strain based on a given crack width when compared to the experimental results. The measured strain in Specimen A shows relatively good visual correlation to the model's estimate. Interestingly, the model's estimates for Specimens A and B are identical despite the fact that, as noted earlier, research has shown that increasing the number of small diameter bars helps to control the crack width ${ }^{25}$. As a result the experimental strain measurements for Specimen B with two $10 \mathrm{M}$ bars are approximately $50 \%$ higher than the model estimates. For Specimen C the experimental FOS strains are a factor of two greater than the estimates based on the crack width. However, once again the Specimen C FOS measurements were higher than expected, suggesting that in reality if the FOS results for Specimen C showed better correlation to the theoretical values in Figure 5, than the strain predictions in Figure 6(a) would also show better correlation to the FOS measurements of Specimen C. One can see from Figure 6(b) that the strain estimates based upon a $\beta$ value of 6 do not agree as closely with the experimental results as the estimates in Figure 6(a) based upon a $\beta$ value of 2. This suggests that the bond between the concrete and the reinforcement is still relatively intact, which is what a lower value of $\beta$ signifies.

Figure 7 presents the relationship between applied load and crack width for the three test specimens. The experimental applied load is the load cell measurement from the experimental test data that corresponds to the DIC crack width measurement. The theoretical applied load was determined by using the calculated strain from equation (1) with a $\beta$ value of 2 as the reinforcement strain. Response 2000 was then used to calculate applied loads corresponding to a given reinforcement strain. The applied load results based upon the use of equation (1) and Response 2000 are plotted in Figure 7 as the theoretical applied loads for each Specimen.

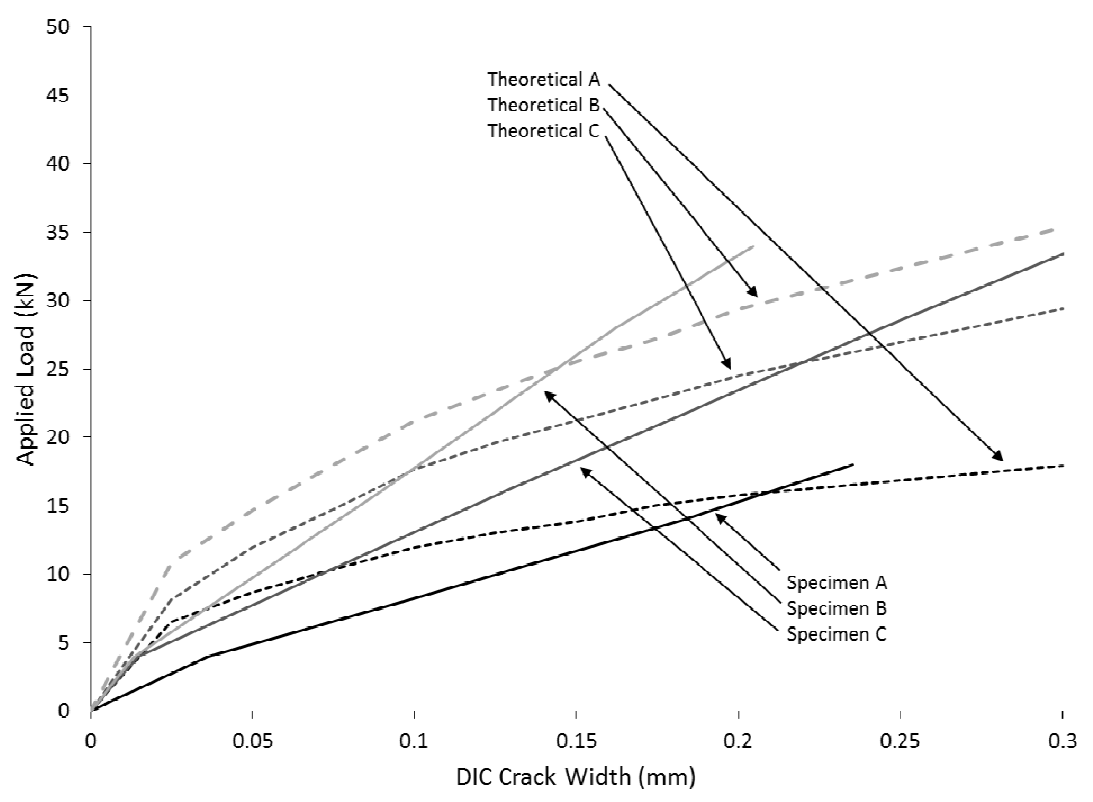

Figure 7. Experimental and theoretical applied load vs. crack width for Specimens A, B, and C.

One can see from Figure 7 that the experimental results show a good visual correlation with the estimated applied loads for Specimens A, B, and C. At all loads Specimen A has the largest cracks both based on the measurements and the model. Though Specimens B and C have the same reinforcement area they have different cracking behavior with Specimen B having the smallest crack widths for a given load. This again matches with the conclusions of Gergely and Lutz $^{25}$ that using a larger number of smaller diameter bars will control cracking better than fewer large diameter bars with the same area. Interestingly, when plotted as a function of applied load, the crack width model proposed by Calvi ${ }^{1}$ appears to match the trends in the experimental data more closely than when the strain measurements are used (Figure 6). 
This is likely because the FOS strain measurements are not used in to evaluate any of the results presented in Figure 7, thus no issues regarding the localized high measured reinforcement strains seen in Figure 5 affect the results of Figure 7. The theoretical prediction for Specimen B provides the highest load for a given crack width while the prediction for Specimen A provides the lowest load, which is the same trend that was seen in the experimental data. The prediction for specimen B is in fact double the prediction for Specimen A since for a given strain in the reinforcement, and thus a given crack width, the force in the reinforcement is double because Specimen B has twice the reinforcement area. Despite the fact that the model shows good correlation between applied load and crack width, it is important to note that further development is necessary regarding the correlation of crack width to internal reinforcement strain. Applied loads and structural element behavior are not always known in the field, thus a more accurate relationship between concrete crack width and internal reinforcement strain would be useful for structural health evaluation applications.

Although this research has provided a proof of concept for the use of sensor technologies to develop relationships between reinforcement strain and crack width, more work is required before the method can be applied practically. A larger experimental database is required involving results from specimens with different reinforcement, cross sectional and loading parameters. In addition, other models that relate crack width to reinforcement stress should be studied since, as was indicated in the discussion, the current model does not differentiate between the number of reinforcement bars used whereas other researchers suggest this is an important parameter.

\section{CONCLUSIONS}

This research program investigated the use of distributed FOS and DIC to measure reinforcement stress and surface crack widths, respectively. Using these sensor technologies it was possible to obtain the maximum reinforcement strain at a crack and the corresponding crack width. The FOS reinforcement strain measurements were compared to theoretical estimates of the strain and the difference between the two values was found to be variable depending on the specimen. The measurements suggested that using multiple small diameter bars led to better crack control than using a single small or large diameter bar at a given stress level. This finding was in agreement with the work of other researchers. Interestingly the results also suggested that bar diameter affected the crack control at a constant stress level although it should be noted that the experimental database is very limited. A model was used to estimate the reinforcement strain based on the measured crack width and it was found to show some correlation to experimental reinforcement strains for a given crack width when the experimental FOS results were close to the theoretical strains. The model also showed good correlation with the measurements when comparing theoretical applied loads to experimental applied loads for a given crack width. Further work is required to determine the impact of a variety of parameters on crack width measurements and to relate these measurements to reinforcement stress.

\section{ACKNOWLEDGEMENTS}

The authors would like to thank the Natural Science and Engineering Research Council of Canada, the Canada Foundation for Innovation, and the Ontario Research Fund for their financial support of this research. The authors would also like to thank Adam Hoag, Jaime Escobar, Neil Porter, and Paul Thrasher for their assistance with the experimental program.

\section{REFERENCES}

[1] Calvi, P. M., "A theory for the shear behaviour of cracks providing a basis for the assessment of cracked reinforced concrete structures," PhD dissertation, Dept. of Civil Eng., University of Toronto, Toronto, Canada (2015).

[2] Scott, R. H. and Beeby, A. W., "Long term tension stiffening effects in concrete," ACI Structural Journal., 102 (1), 31-39 (2005).

[3] Masukawa, J., "Degradation of Shear Performance of Beams due to Bond Deterioration and Longitudinal Bar Cutoffs," Ph.D. Dissertation, University of Toronto (2012).

[4] Hoult, N., and Soga, K., "Sensing solutions for assessing and monitoring tunnels," Sensor Technologies for Civil Infrastructures: Applications in Structural Health Monitoring, 2, 309-346 (2014).

[5] Gebremichael, Y.M., Li, W., Meggitt, B.T., Boyle, W.J.O., Grattan, K.T.V., McKinley, B., Boswell, L.F., Aarnes, K.A., Aasen, S.E., Tynes, B., Fonjallaz, Y., and Triantafillou, T., "A Field Deployable, Multiplexed Bragg Grating Sensor System Used in an Extensive Highway Bridge Monitoring Evaluation Tests," IEEE Sensors Journal. , 5(3), 510-519 (2005). 
[6] Bastianini, F., Matta, F., Rizzo, A., Galati, N., and Nanni, A., "Overview of recent bridge monitoring applications using distributed Brillouin fiber optic sensors," J. Nondestr. Test, 12(9), 269-276 (2007).

[7] Mohamad, H., Soga, K., Pellew, A., and Bennett, P. J., "Performance monitoring of a secant-piled wall using distributed fiber optic strain sensing," J. Geotech. Geoenviron. Eng., 10.1061/(ASCE)GT.1943-5606.0000543, 1236-1243 (2011).

[8] Henault, J. M., Salin, J., Moreau, G., Delepine-Lesoille, S., Bertand, J., Taillade, F.,Quiertant, M. and Benzarti, K., "Qualification of a truly distributed fiber optic technique for strain and temperature measurements in concrete structures," In EPJ Web of Conferences 12, 3004 (2011).

[9] Kreger, S.T., Gifford, D.K., Froggatt, M.E., Sang, A.K., Duncan, R.G., Wolfe, M.S. and Soller, B.J., "Highresolution extended distance distributed fiber-optic sensing using rayleigh backscatter," In The 14th International Symposium on: Smart Structures and Materials \& Nondestructive Evaluation and Health Monitoring, 65301R65301R (2007).

[10] Villalba, S., and Casas, J. R., "Application of optical fiber distributed sensing to health monitoring of concrete structures.” Mech. Syst. Sig. Process., 39(1-2), 441-451 (2013).

[11] Regier, R., "Application of fibre optics on reinforced concrete structures to develop a structural health monitoring technique," MASc. Thesis, Queen's University Department of Civil Engineering (2013).

[12] Regier, R. and Hoult, N., "Distributed Strain Behavior of a Reinforced Concrete Bridge: Case Study," J. Bridge Eng., 19(12), 05014007 (2014).

[13] Regier, R. and Hoult, N., "Concrete deterioration detection using distributed sensors," Proceedings of the ICE Structures and Buildings, 168(2), $118-126$ (2014).

[14] Lecompte, D, Vantomme, J, and Sol, H., "Crack Detection in a Concrete Beam using Two Different Camera Techniques," Structural Health Monitoring, 5(1), 59-68 (2006).

[15] Destrebecq, J. F., Toussaint, E., and Ferrier, E., "Analysis of Cracks and Deformations in a Full Scale Reinforced Concrete Beam Using a Digital Image Correlation Technique,” Experimental Mechanics. 51(6), 879-890 (2010).

[16]Ferreira, M. D., Venturini, W. S., and Hild, F., "On the analysis of notched concrete beams: From measurement with digital image correlation to identification with boundary element method of a cohesive model," Engineering Fracture Mechanics, 78(1), 71-84 (2011).

[17] Jason, L., Torre-Casanova, A., Davenne, L., and Pinelli, X., "Cracking behavior of reinforced concrete beams: experiment and simulations on the numerical influence of the steel-concrete bond." International Journal of Fracture, 180(2), 243-260 (2013).

[18] White, D. J., Take, W. A., and Bolton, M. D., "Soil deformation measurement using particle image velocimetry (PIV) and photogrammetry," Géotechnique, 50(7), 619-631 (2003).

[19] Murray, C., Hoag, A. Hoult, N.A. and Take, W.A., "Field Monitoring of a Bridge using Digital Image Correlation," ICE Bridge Engineering, 10.1680/bren.13.00024 (2014).

[20] Hoult, N. A., Take, W. A., Lee, C., and Dutton, M., "Experimental Accuracy Two Dimensional Strain Measurements using Digital Image Correlation," Engineering Structures, 46, 718-726 (2013).

[21] Dutton, M., Take, W., and Hoult, N.,"Curvature Monitoring of Beams Using Digital Image Correlation,” J. Bridge Eng., 19(3), 05013001 (2014).

[22] Shima, H., Chou, L. and Okamura, H., "Micro and macro models for bond in reinforced concrete," Journal of the Faculty of Engineering, The University of Tokyo (B), 39(2), 133-194 (1987).

[23] Maekawa, K., Pimanmas, A. and Okamura, H., [Nonlinear Mechanics of Reinforced Concrete], Spon Press, London (2003).

[24]Bentz, E. C., "Sectional Analysis of Reinforced Concrete Members," PhD dissertation, Dept. of Civil Eng., University of Toronto, Toronto, Canada (2000).

[25] Gergely, P., and Lutz, L. A., "Maximum Crack Width in Reinforced Concrete Flexural Members," Causes, Mechanism, and Control of Cracking in Concrete, SP-20, R. E. Philleo, ed., American Concrete Institute, Farmington Hills, Mich., 87-117 (1968). 